\title{
REMOTE SENSING OF ATMOSPHERIC METHANE WITH SIMULTANEOUS RANGING USING CHIRPED LASER DISPERSION SPECTROSCOPY
}

\author{
Genevieve Plant ${ }^{1}$, Andreas Hangauer ${ }^{1,2}$, Gerard Wysocki ${ }^{\text {* }}$ \\ ${ }^{1}$ Electrical Engineering, Princeton University, NJ 08544, USA, *Email: gwysocki@princeton.edu \\ ${ }^{2}$ Currently: Siemens, Munich, Germany
}

\begin{abstract}
We present a new sensing technology that allows for simultaneous sensing and ranging using chirped laser dispersion spectroscopy (CLaDS). In conjunction with previous works demonstrating the effectiveness of CLaDS for remote sensing, this new configuration yields spectroscopic and ranging information from a single measurement, and is implemented for continuous, multi-path detection of atmospheric methane.
\end{abstract}

\section{INTRODUCTION}

In remote sensing of atmospheric trace gases, knowledge of the path length is crucial to accurate extraction of the species concentration. However, in most case, this optical path length must be known beforehand or measured separately. Currently, the standard technique for atmospheric ranging and sensing is lidar (light radar), where pulsed light probes the target environment. The timing of the returned backscatter is used to extract the path length, and the received intensity is analyzed to determine the path-integrated concentration. We present an alternative, continuous wave $(\mathrm{CW})$, approach based on the fundamentals of chirped laser dispersion spectroscopy (CLaDS). A proof-of-concept study has shown that both species concentration and path length can be extracted from a single, CW measurement. Development of a system that combines this $\mathrm{CW}$ sensing and ranging feature, with a CLaDS system that monitors atmospheric methane will be presented.

\section{CHIRPED LASER DISPERSION SPECTRSOCOPY FOR REMOTE ATMOSHPERIC SENSING}

Chirped laser dispersion spectroscopy is a new technique that probes the optical dispersion induced when probe laser light interacts with a targeted species [1]. When the laser frequency coincides with a molecular transition, light is absorbed, however, in addition the light also experiences dispersion which is related to resonant absorption via the Kramers-Kronig relationship. Although the sensed gas parameters are extracted from the spectral measurement in a way very similar to that of absorption-based techniques, CLaDS has unique properties that make it particularly well suited for remote sensing configurations. These include CLaDS signal linearity with concentration when implemented in a single-sideband configuration [2], immunity to intensity fluctuations [1], and possible harmonic detection using chirp-modulated CLaDS (CMCLaDS) [3]. We have already demonstrated the operation of a CLaDS instruments for the application of atmospheric methane monitoring [4]. The developed instrument was tested in a wetland to target natural emissions of methane. Multiple paths were probed using a motorized mirror to cycle through the various path locations (defined by retro-reflector placement) [5]. However, like most systems, the optical length for each path was initially measured separately and kept constant throughout the campaign. Therefore the new capability of simultaneous ranging adds an important functionality that will enable dynamic variable-path chemical sensing.

\section{SIMULTANEOUS SENSING AND RANGING}

We have recently demonstrated an upgraded CLaDS technique that performs simultaneous chemical sensing and ranging [6]. The ranging measurement is performed using a $\mathrm{CW}$ radar method implemented in the form of the frequency chirped modulation sidebands (chirp 2 of frequency $\Omega$ in Fig. 1), which are used in conventional CLaDS to probe the target molecular transition. As a result of the propagation delay between the transmitter and the receiver, this additional chirp causes a frequency shift to the $0^{\text {th }}$ harmonic signal equal to $\tau_{g}: S_{\square}$ (where $\tau_{g}$ [s] is the total group delay and $S_{\square}[\mathrm{Hz} / \mathrm{s}]$ is the carrier chirp rate), while the molecular signals (CM-CLaDS spectra) demodulated at higher harmonics are not affected. Thus extraction of the path-integrated concentration is performed using the same algorithms as in conventional CLaDS systems. 


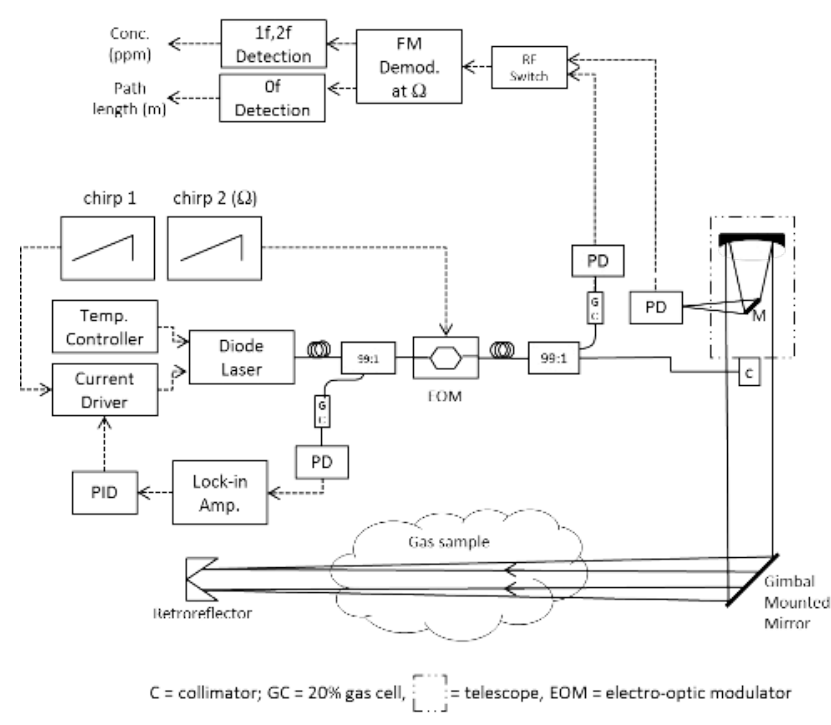

Figure 1. System diagram for remote detection of atmospheric methane with simultaneous ranging information. The major difference with respect to a conventional CLaDS is the addition chirp of modulation sidebands ("chirp2").

Results from proof-of-concept testing show the path length and gas signal measured for multiple path lengths (Fig.2). The path length, $L$, is determined using the frequency offset, $\Delta f$ (shown in the top plot of Fig.2, and the carrier chirp rate.

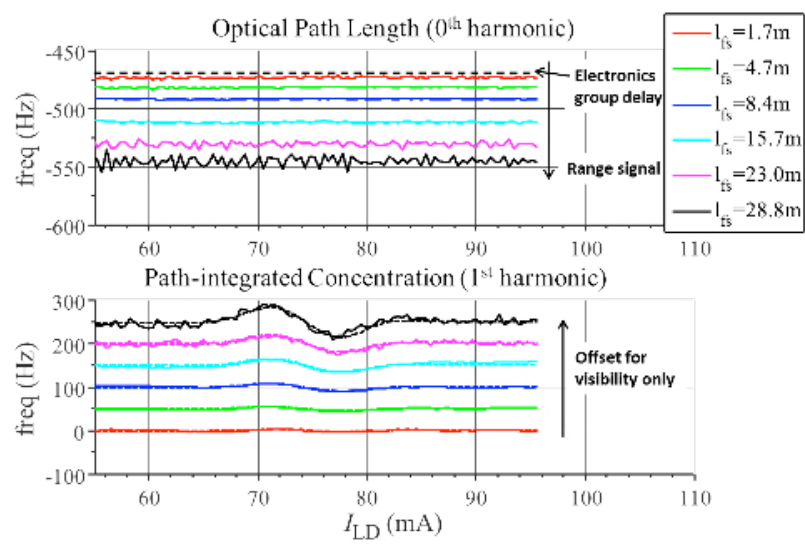

Figure 2. Proof of concept results demonstrating the offset of the $0^{\text {th }}$ harmonic signals containing the pathlength information, while the $1^{\text {st }}$ harmonic provided the CLaDS signal used for methane concentration retrieval [6].

The relationship follows as $L=\left(\Delta f-\Delta f_{o f s}\right) /\left(S_{\square} \cdot c\right)$, where $\Delta f_{\text {ofs }}$ and $c$ are the frequency offset produced by the delay of the generation electronics (a constant) and the speed of light, respectively. Integration of this range-retrieval method into the second generation instrument for atmospheric methane sensing allows for increased flexibility on path selection as well as the possibility for implementation of moving targets or hard targets at unknown distances.

\section{MULTI-PATH MEASUREMENTS}

Due to the chirp of the carrier frequency, $\square$, different beatnote frequencies will be generated for different path lengths. Thus, multiple paths can be sensed simultaneously while the corresponding signals are extracted using straightforward filtering. This method of simultaneous multi-path sensing has been demonstrated so far only in a fiber-based multinode sensor systems [7], and in this work a first open-path implementation into our remote methane sensor will be discussed.

\section{CONCLUSIONS}

Using the foundation of chirped laser dispersion spectroscopy, a simultaneous ranging and sensing technique has been developed using a continuouswave laser source. Implementation into a methane sensor for multi-path atmospheric monitoring applications will be discussed in details.

\section{ACKNOWLEDGEMENTS}

The authors would like to acknowledge financial support by the NSF CAREER award CMMI-0954897 and by the NSF ERC MIRTHE award EEC-0540832

\section{REFERENCES}

[1] G. Wysocki and D. Weidmann, "Molecular dispersion spectroscopy for chemical sensing using chirped mid-infrared quantum cascade laser," Optics Express 18, 26123-26140 (2010). [2] M. Nikodem and G. Wysocki, "Measuring optically thick molecular samples using chirped laser dispersion spectroscopy," Optics Letters 38, 3834-3837 (2013).

[3] M. Nikodem, D. Weidmann, and G. Wysocki, "Chirped laser dispersion spectroscopy with harmonic detection of molecular spectra," Applied Physics B 109, 477-483 (2012).

[4] M. Nikodem, G. Plant, D. Sonnenfroh, and G. Wysocki, "Open-path sensor for atmospheric methane based on chirped laser dispersion spectroscopy," Applied Physics B, 1-7 (2014).

[5] G. Plant, Y. Tian, T. Wang, and G. Wysocki, "Improved Signal Processing for Distributed Sensing Network based on Chirped Laser Dispersion Spectroscopy," in CLEO: 2014, (Optical Society of America, 2014), SM3E.2.

[6] A. Hangauer and G. Wysocki, "Simultaneous ranging and remote sensing utilizing chirped laser dispersion spectroscopy," in CLEO: 2014 Postdeadline Paper Digest, OSA Technical Digest (online) (Optical Society of America, 2014), STh5C.5.

[7] G. Plant, A. Hangauer, Y. Tian, T. Wang, and G. Wysocki, "Methane Leak Detection Sensor Network based on Fibercoupled Chirped Laser Dispersion Spectroscopy," in Light, Energy and the Environment, (Optical Society of America, 2014), ETu4A.2. 